\title{
Synthesis of Ru(II) Complexes of N-Heterocyclic Carbenes and Their Promising Photoluminescence Properties in Water
}

\author{
Seung Uk Son, ${ }^{\dagger}$ Kang Hyun Park, ${ }^{\dagger}$ Young-Shin Lee,${ }^{\dagger}$ Bo Yun Kim, ${ }^{\dagger}$ Cheol Ho Choi, ${ }^{\ddagger}$ \\ Myoung Soo Lah, ${ }^{\S}$ Yun Hee Jang, ${ }^{\dagger}$ Du-Jeon Jang, ${ }^{\dagger}$ and Young Keun Chung ${ }^{\dagger} *$
}

School of Chemistry and Center for Molecular Catalysis, Seoul National University, Seoul 151-747,

Korea. Department of Chemistry, College of Natural Sciences, Kyungpook National University,

Daegu 702-701, Korea. Department of Chemistry, College of Science, Hanyang University, Ansan, Kyunggi-Do 425-791, Korea

ykchung@plaza.snu.ac.kr

\section{Experimental Section}

\section{Characterization of New Compounds.}

1) General. All reactions were conducted under nitrogen using standard Schlenk-type flasks. Workup procedures were done in air. Reagents were purchased from Aldrich Chemical Co. and Strem Chemical Co. and were used as received. ${ }^{1} \mathrm{H}$ NMR spectra were obtained with a Bruker 300 spectrometer. Elemental analyses were done at National Center for Inter-University Research Facilities, Seoul National University. High Resolution Mass was done at Korea Basic Science Institute.

2) Ligands $\mathbf{L} \mathbf{1}$ and $\mathbf{L} \mathbf{2}$ were synthesized by literature methods. ${ }^{1}$

3) Synthesis of 1. A mixture of $\mathbf{L 1}(0.415 \mathrm{~g}, 1.4 \mathrm{mmol})$ and ruthenium(III) chloride $(0.100 \mathrm{~g}, 0.48$ mmol) in $10 \mathrm{~mL}$ of 1,2-ethanediol was refluxed for $4 \mathrm{~h}$. Methanol $(100 \mathrm{~mL})$ and water $(30 \mathrm{~mL})$ were added to the reaction mixture. A solution of $2.0 \mathrm{~g}$ of $\mathrm{NH}_{4} \mathrm{PF}_{6}$ dissolved in water/methanol (10:3) was added. The resulting precipitates were filtered, rinsed with water, and dried. Yield: $0.31 \mathrm{~g}(0.36 \mathrm{mmol}$, 75\%); ${ }^{1} \mathrm{H}$ NMR $\left(\mathrm{CD}_{3} \mathrm{NO}_{2}\right): \delta 8.06(\mathrm{t}, 8.2 \mathrm{~Hz}, 1 \mathrm{H}), 7.98-7.88(\mathrm{~m}, 8 \mathrm{H}), 7.79(\mathrm{~d}, 8.2 \mathrm{~Hz}, 2 \mathrm{H}), 7.72(\mathrm{~d}$, $5.8 \mathrm{~Hz}, 1 \mathrm{H}), 7.26(\mathrm{t}, 6.8 \mathrm{~Hz}, 1 \mathrm{H}), 7.22(\mathrm{dd}, 4.4,2.2 \mathrm{~Hz}, 2 \mathrm{H}), 7.14$ (d, $2.3 \mathrm{~Hz}, 1 \mathrm{H}), 7.05$ (t, $7.1 \mathrm{~Hz}, 2$ $\mathrm{H}), 3.30(\mathrm{~s}, 3 \mathrm{H}), 3.03$ (s, $6 \mathrm{H}) \mathrm{ppm}$; Anal. Calcd. for $\mathrm{C}_{27} \mathrm{H}_{27} \mathrm{~N}_{9} \mathrm{RuP}_{2} \mathrm{~F}_{12}$ : C, 37.34; H, 3.13; N, 14.51 . Found: C, 37.46; H, 3.18; N, 14.51.

4) Synthesis of $\mathbf{2}\left(\mathrm{PF}_{6}^{-}\right)$. Complex $\mathbf{2}\left(\mathrm{PF}_{6}^{-}\right)$was synthesized by the same method as the synthesis of $\mathbf{1}$ except 2( $\left.\mathrm{PF}_{6}{ }^{-}\right)$equiv $\mathbf{L 2}$ instead L1. Yield: $82 \% ;{ }^{1} \mathrm{H} \mathrm{NMR}\left(\mathrm{CD}_{3} \mathrm{NO}_{2}\right): \delta 8.25$ (t, $\left.8.4 \mathrm{~Hz}, 2 \mathrm{H}\right), 8.01$ (d, $2.2 \mathrm{~Hz}, 4 \mathrm{H}), 7.87(\mathrm{~d}, 8.2 \mathrm{~Hz}, 4 \mathrm{H}), 6.99$ (d, $2.1 \mathrm{~Hz}, 4 \mathrm{H}), 2.71$ (s, $12 \mathrm{H}) \mathrm{ppm}$; Anal. Calcd. for $\mathrm{C}_{26} \mathrm{H}_{26} \mathrm{~N}_{10} \mathrm{Ru}_{1} \mathrm{P}_{2} \mathrm{~F}_{12}$ : C, 35.91; H, 3.01; N, 16.11. Found: C, 36.36; H, 3.05; N, 16.27. 
5) Synthesis of $2\left(\mathrm{BPh}_{4}{ }^{-}\right)$. Complex $2\left(\mathrm{BPh}_{4}{ }^{-}\right)$were synthesized by the same method as the synthesis of 2( $\left.\mathrm{PF}_{6}^{-}\right)$except $\mathrm{NaBPh}_{4}$ instead of $\mathrm{NH}_{4} \mathrm{PF}_{6}$. Yield: $80 \%$; ${ }^{1} \mathrm{H}$ NMR (acetone- $\left.\mathrm{d}_{6}\right): \delta 8.32(\mathrm{t}, 8.2 \mathrm{~Hz}, 2 \mathrm{H}$ ), 8.30 (d, 2.2 Hz, 4 H), 8.08 (d, 8.0 Hz, 4 H), 7.33 (m, 16 H), 7.18 (d, 2.2 Hz, 4 H), 6.90 (t, 7.4 Hz, 16 $\mathrm{H}$ ), 6.76 (t, 7.0 Hz, $8 \mathrm{H}$ ), 2.74 (s, 12H) ppm; Anal. Calcd. for $\mathrm{C}_{74} \mathrm{H}_{66} \mathrm{~N}_{10} \mathrm{~B}_{2} \mathrm{Ru}_{1}$ : C, 72.97; H, 5.46; N, 11.50. Found: C, 72.98; H, 5.47; N, 11.48 .

6) Synthesis of $\mathbf{2}\left(\mathrm{Br}^{-}\right)$. Complex $\mathbf{2}(\mathrm{Br})$ was synthesized by the same method as the synthesis of $\mathbf{2}\left(\mathrm{PF}_{6}{ }^{-}\right.$ ) without using other counteranion source. Yield: $65 \% ;{ }^{1} \mathrm{H}$ NMR $\left(\mathrm{D}_{2} \mathrm{O}\right): \delta 8.25(\mathrm{t}, 8.4 \mathrm{~Hz}, 2 \mathrm{H}), 8.10(\mathrm{~s}$, $4 \mathrm{H}), 7.93(\mathrm{~d}, 8.3 \mathrm{~Hz}, 4 \mathrm{H}), 7.05$ (s, $4 \mathrm{H}), 2.68$ (s, 12H) ppm; Anal. Calcd. for $\mathrm{C}_{26} \mathrm{H}_{26} \mathrm{~N}_{10} \mathrm{RuBr}_{2}$ : C, 42.23; H, 3.54; N, 18.94. Found: C, 42.30; H, 4.00; N, 18.89.

2. Method of Crystal Growth and X-ray Analysis. Single crystals of $2\left(\mathrm{BPh}_{4}{ }^{-}\right)$suitable for $\mathrm{X}$-ray diffraction study were grown by slow diffusion of diethy ether to the nitromethande solution of $2\left(\mathrm{BPh}_{4}{ }^{-}\right)$ at room temperature. X-ray data for single crystals were collected on a Bruker SMART X-ray diffractometer at room temperature using graphite-monochromated Mo K $\alpha$ radiation $(\lambda=0.71073 \AA)$. The structures were solved by direct methods (SHELXS-97), and refined against all $\mathrm{F}^{2}$ data (SHELXS97). All non-hydrogen atoms were refined with anisotropic thermal parameters and the hydrogen atoms were treated as idealized contributions.

3. UV/Vis Experiment. The UV/vis absorption spectra of the complexes were obtained at the concentrations of $2.33 \times 10^{-5} \mathrm{M}$ in acetonitrile or $\mathrm{H}_{2} \mathrm{O}$ using a Beckman Du-650 spectrometer.

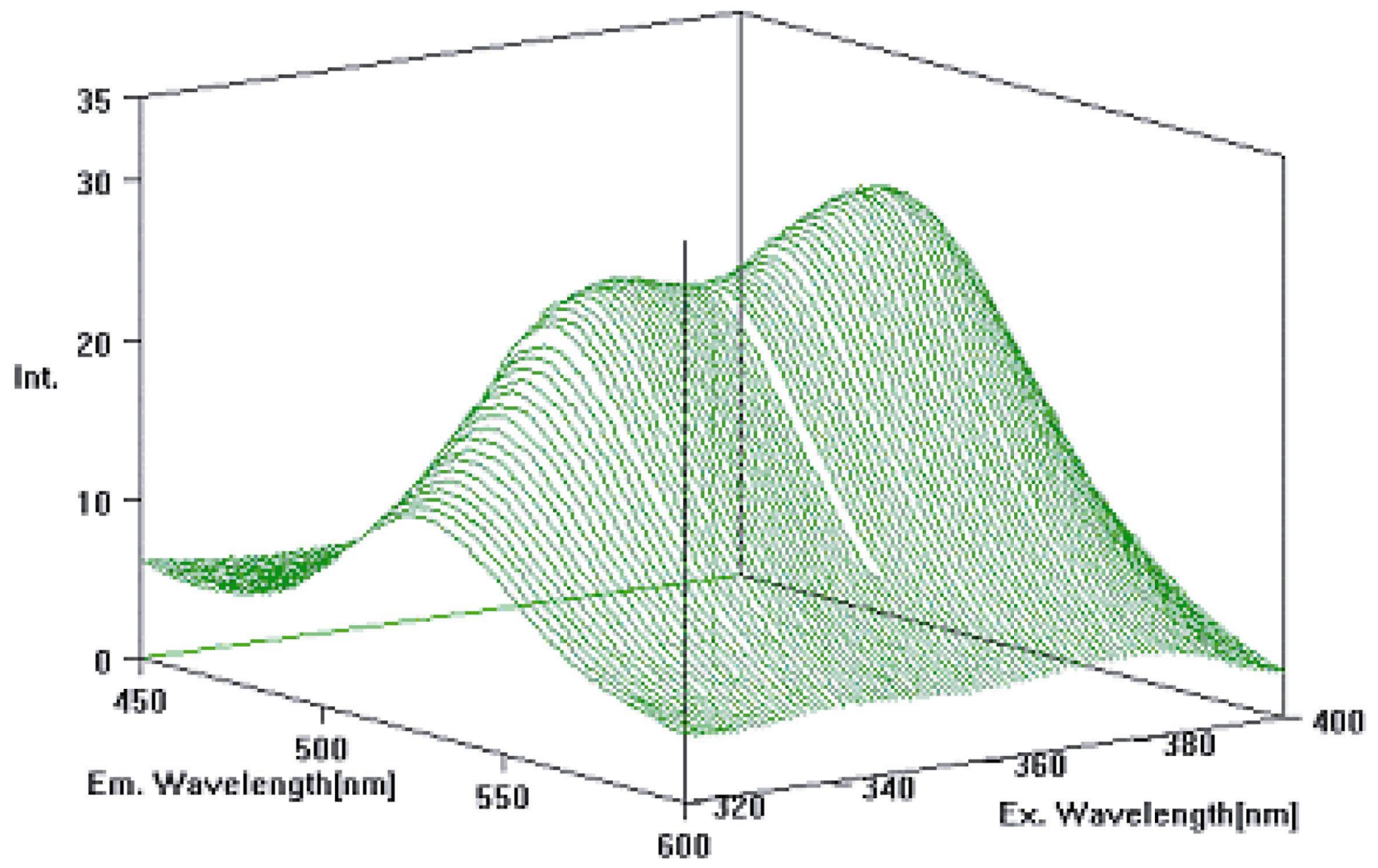




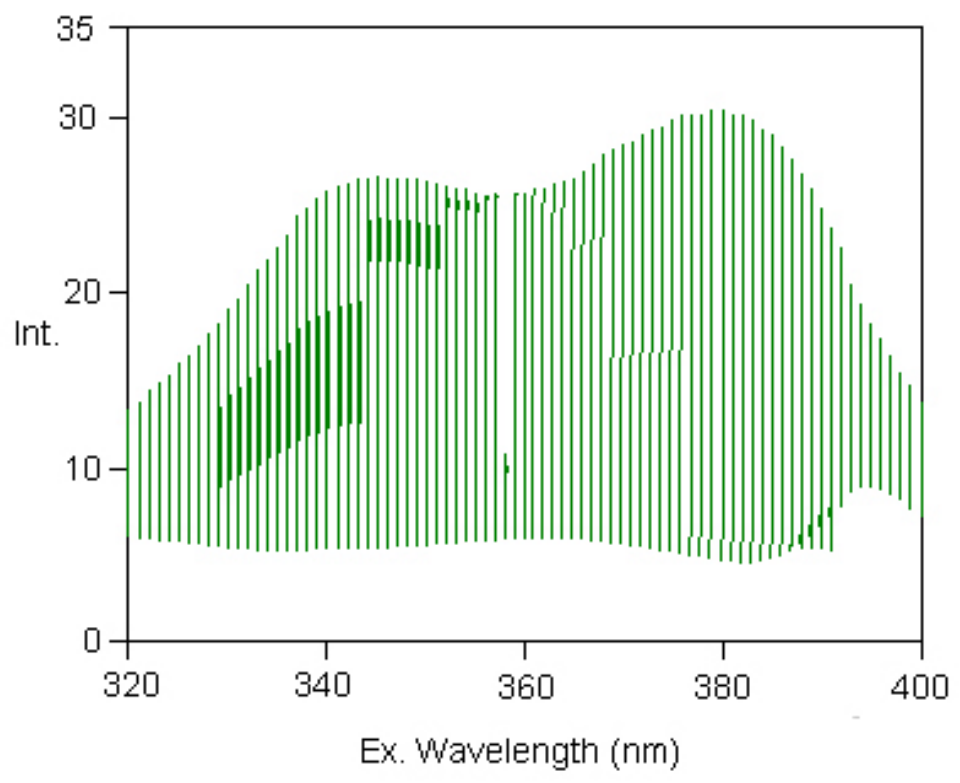

Figure S1. A 3D emission spectrum of $2\left(\mathrm{PF}_{6}^{-}\right)$in acetonitile.

4. Cyclic Voltammetry Experiment. Cyclic voltammograms were obtained with a BAS CV-50W voltametric analyzer. The working electrode was glassy carbon, with an $\mathrm{Ag} / \mathrm{AgCl}$ electrode separated from the test solution by a salt-bridge containing the solvent and supporting electrolyte. The auxiliary electrode was a platinum wire. Solutions studied were $1 \mathrm{mM}$ in solute and $0.1 \mathrm{M}$ in tetrabutylammonium hexafluorophosphate as a supporting electrolyte. HPLC grade dichloromethane was used. A dinitrogen atmosphere was performed over the solution during the experiment. The scan rate was $100 \mathrm{mV} / \mathrm{s}$. 


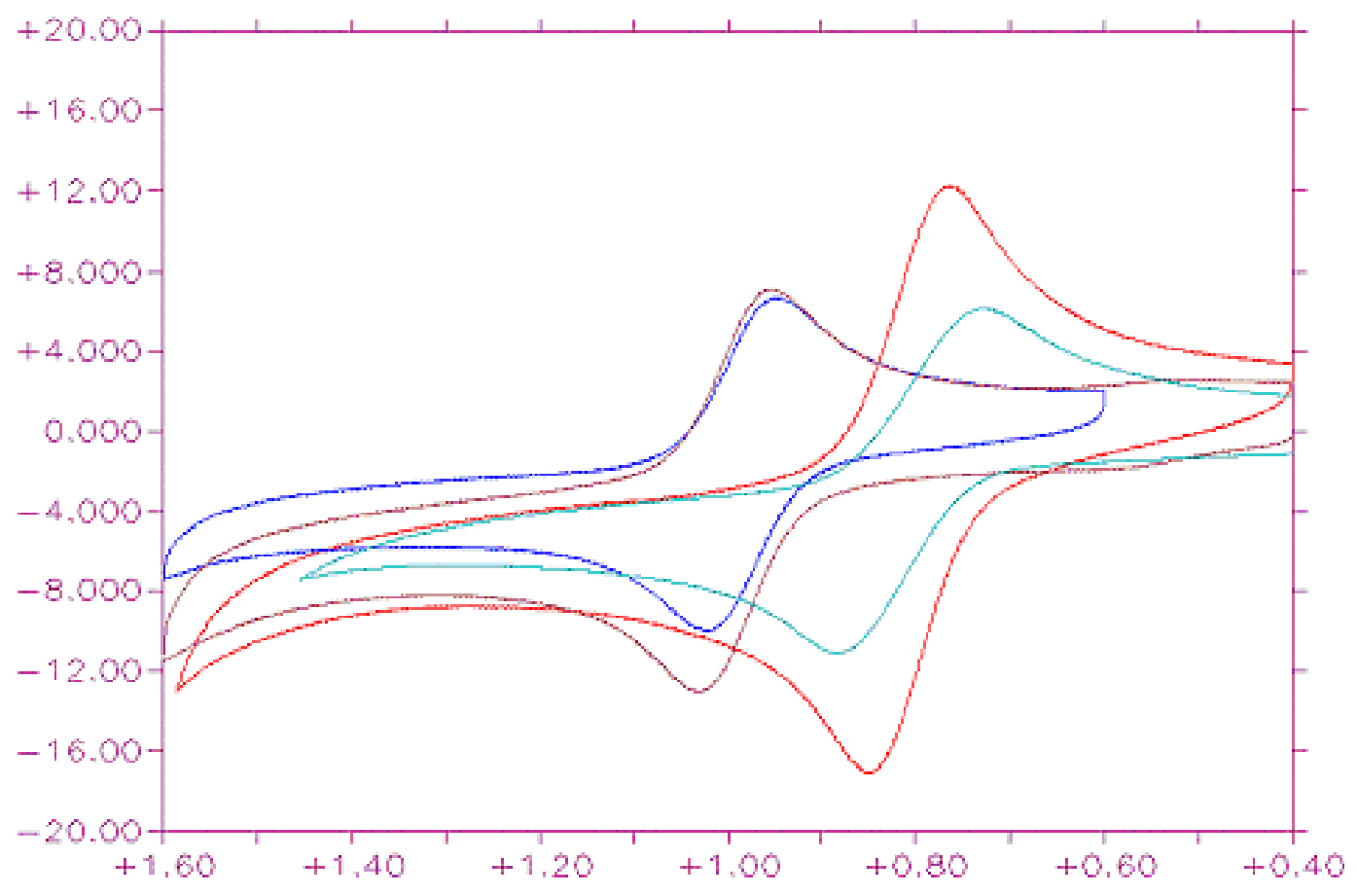

Figure S2. Cyclic Voltammetry study in acetonitrile with $0.1 \mathrm{M}$ TBAP. The scan rate was $100 \mathrm{mV} / \mathrm{s}$.

Table S1. Half Potential of Oxidation Peaks of Various $\mathrm{Ru}^{2+}$ Complexes

\begin{tabular}{ccccc}
\hline half potential $^{*}$ & $\mathrm{Ru}(\text { by })_{3}{ }^{2+}$ & $\mathrm{Ru}(\text { terpy })_{2}{ }^{2+}$ & $\mathbf{1}$ & $\mathbf{2}\left(\mathrm{PF}_{6}{ }^{-}\right)$ \\
\hline $\mathrm{Ru}^{2+} / \mathrm{Ru}^{3+}(\mathrm{V})$ & 0.996 & 0.979 & 0.806 & 0.807 \\
\hline *. Ferrocene: $+0.0675 \mathrm{~V}$ & & &
\end{tabular}

5. Measurements of Luminescence Spectra and Kinetics. Luminescence spectra were obtained by using a fluorometer consisting of 75-W Xe-lamp (Acton Research, XS 432) and two 0.30-m monochromators (Acton Research, Spectropro 300). The third harmonic pulses (355 nm) of a Qswitched Nd: YAG laser (Quantel, Brilliant) operated at $10 \mathrm{~Hz}$ were used to excite samples for luminescence kinetics measurements at room temperature. Wavelength-selected emission was detected by a photomultiplier tube, whose output was transmitted to a digital oscilloscope (Lecroy, Wavepro 950). Luminescence kinetic constants were extracted by fitting the measured profiles to the computersimulated exponential curves.

6. Calculation. Full geometry optimizations were performed with Gaussian 98 program. ${ }^{2}$ Lee, Yang, and Parr's $(\mathrm{LYP})^{3}$ correlation functional, and Becke's three parameter hybrid (B3) ${ }^{4}$ exchange 
functionals using LanL2DZ effective core potential were used for the gradient-corrected density approximation.

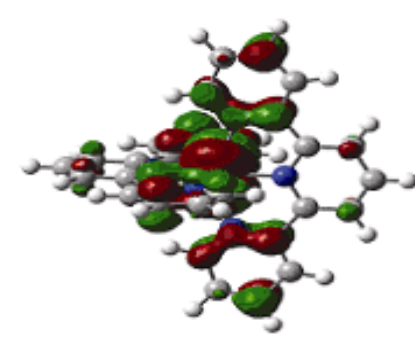

номо(129)

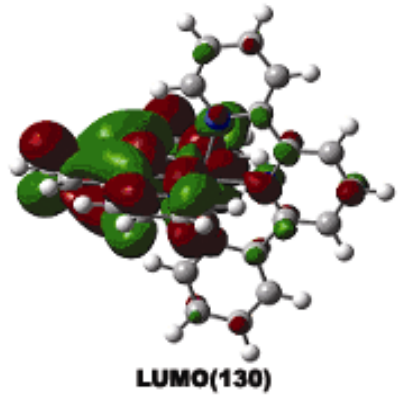

(a)

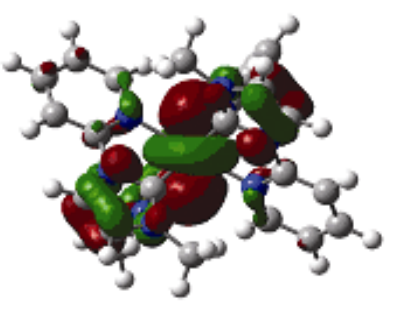

номо(133)

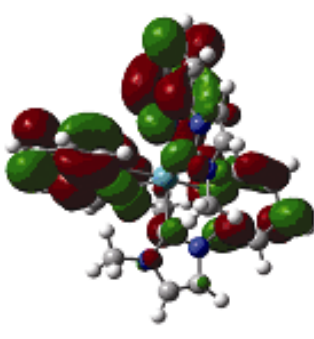

LUMO(134)

(b)

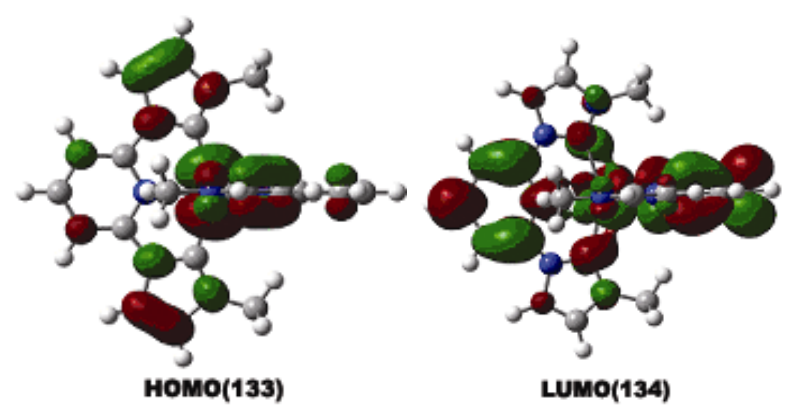

(c)

Figure S3. (a) HOMO and (b) LUMO density surfaces for Ru(terpy) ${ }_{2}{ }^{2+}(\mathrm{a}), \mathbf{1}(\mathrm{b})$, and $\mathbf{2}\left(\mathrm{PF}_{6}{ }^{-}\right)(\mathrm{c})$.

Table S2. Orbital energies in Hartree and orbital number as obtained with B3LYP/6-31G*.

\begin{tabular}{|c|c|c|c|c|c|}
\hline \multicolumn{2}{|l|}{$\mathrm{Ru}(\text { terpy })_{2}{ }^{2+}$} & \multicolumn{2}{|l|}{1} & \multicolumn{2}{|l|}{$2\left(\mathrm{PF}_{6}^{-}\right)$} \\
\hline \multicolumn{2}{|c|}{$\begin{array}{l}\text { Orbitals } 127,128 \text {, and } 129 \\
\text { are dxy, dyz, and dzx }\end{array}$} & \multicolumn{2}{|c|}{$\begin{array}{l}\text { Orbitals } 131,132 \text {, and } 133 \\
\text { are dxy, dyz, and dzx }\end{array}$} & \multicolumn{2}{|c|}{$\begin{array}{l}\text { Orbitals } 131,132, \text { and } 133 \\
\text { are dxy, dyz, and dzx. }\end{array}$} \\
\hline \multicolumn{2}{|c|}{$\begin{array}{l}\text { Orbitals } 130-137 \text { have a } \\
\text { ligand character. }\end{array}$} & \multicolumn{2}{|c|}{$\begin{array}{l}\text { Orbitals } 134-139 \text { have a } \\
\text { ligand character. }\end{array}$} & \multicolumn{2}{|c|}{$\begin{array}{l}\text { Orbitals } 134-139 \text { have a } \\
\text { ligand character. }\end{array}$} \\
\hline-0.41447 & 127 & -0.40368 & 131 & -0.40174 & 131 \\
\hline-0.41446 & 128 & -0.40146 & 132 & -0.40173 & 132 \\
\hline-0.41187 (HOMO) & 129 & $-0.39463(\mathrm{HOMO})$ & 133 & -0.39614 (HOMO) & 133 \\
\hline-0.28091 (LUMO) & 130 & -0.25219 (LUMO) & 134 & -0.25422 (LUMO) & 134 \\
\hline-0.28091 & 131 & -0.24932 & 135 & -0.25421 & 135 \\
\hline-0.27591 & 132 & -0.24814 & 136 & -0.24219 & 136 \\
\hline
\end{tabular}




\begin{tabular}{|c|c|c|c|c|c|}
\hline-0.27362 & 133 & -0.23578 & 137 & -0.24155 & 137 \\
\hline-0.24439 & 134 & -0.23268 & 138 & -0.18971 & 138 \\
\hline-0.24060 & 135 & -0.23010 & 139 & -0.18969 & 139 \\
\hline-0.24060 & 136 & $-0.19195\left(\mathrm{~d} \mathrm{z}^{2}\right)$ & 140 & $-0.18100\left(\mathrm{~d} \mathrm{z}^{2}\right)$ & 140 \\
\hline-0.23665 & 137 & $-0.18150\left(d x^{2}+y^{2}\right)$ & 141 & $-0.17671\left(\mathrm{dx}^{2}+\mathrm{y}^{2}\right)$ & 141 \\
\hline$-0.21873\left(\mathrm{~d} \mathrm{z}^{2}\right)$ & 138 & & & & \\
\hline$-0.21251\left(\mathrm{dx}^{2}+\mathrm{y}^{2}\right)$ & 139 & & & & \\
\hline \multicolumn{2}{|c|}{$\begin{array}{l}\text { Energy gap between } \\
\text { HOMO and LUMO: } 3.56 \\
\text { eV }\end{array}$} & \multicolumn{2}{|c|}{$\begin{array}{l}\text { Energy gap between } \\
\text { HOMO and LUMO: } 3.86 \\
\text { eV }\end{array}$} & \multicolumn{2}{|c|}{$\begin{array}{l}\text { Energy gap between HOMO } \\
\text { and LUMO: } 3.86 \mathrm{eV}\end{array}$} \\
\hline
\end{tabular}

\section{Refrence}

1 (a) Chen, J. C. C.; Lin, I. J. B. J. Chem. Soc., Dalton Trans. 2000, 839. (b) Grunedemann, S.;

Kovacevic, A.; Albrecht, M.; Faller, J. W.; Crabtree, R. H. J. Am. Chem. Soc. 2002, 124, 10473.

2. Frisch, M. J. et al. Gaussian 98, Gaussian, Inc., Pittsburgh PA, 1995.

3. Lee, C.; Yang, W.; Parr, R. G. Phys. Rev. B37 1988, 785.

4. Becke, A. D. J. Chem. Phys. 1993, 98,1372. 